\title{
Seismic Performance and Application of Sandwiched Buckling-Restrained Braces and Dual-Core Self-Centering Braces
}

\author{
Chung-Che Chou ${ }^{1,2}$, Ping-Ting Chung ${ }^{1} \&$ Ying-Chuan Chen ${ }^{1}$ \\ ${ }^{1}$ Dept. of Civil Engineering, National Taiwan University, \\ No. 1, Roosevelt Road Section 4, Daan District, Taipei City 10617, Taiwan \\ ${ }^{2}$ Research Fellow, National Center for Research on Earthquake Engineering, \\ 200, Sec. 3, HsinHai Rd., Taipei 10668, Taiwan \\ Email: cechou@ntu.edu.tw
}

\begin{abstract}
This paper first presents cyclic test results and the application of the proposed sandwiched buckling-restrained brace (BRB). The proposed BRB can be easily disassembled in the field. This provides an opportunity for inspection of the core after a large earthquake. The mechanics and cyclic behavior of a novel steel dual-core self-centering brace (SCB) are then proposed and introduced, followed by the testing of a dual-core SCB in order to evaluate its cyclic performance. Both braces achieve an excellent target lateral drift performance of up to $2.5 \%$, thus satisfying the seismic requirement by the AISC Seismic Provisions 2010.
\end{abstract}

Keywords: cyclic test; dual-core self-centering brace (SCB); sandwiched bucklingrestrained brace (BRB).

\section{$1 \quad$ Introduction}

This paper presents cyclic test results of two energy-dissipating braces that are used for enhancing earthquake-resistance of structures. The first brace is called a sandwiched buckling-restrained brace (BRB). This is comparable to conventional BRBs that have a steel core inserted into a restraining member, however in this case, bolts are used to sandwich a core between a pair of restraining members, which enables fast assemblage and provides opportunities for inspecting the core after large earthquakes [1].

The second brace is a novel steel dual-core self-centering brace (SCB), which was developed in Taiwan by applying post-tensioning (PT) technology in a single brace to reduce the residual drift of structures. A novel dual-core SCB $[2,3]$ consists of conventional steel bracing members, energy dissipative devices, and two sets of tensioning elements that are in a parallel arrangement to double the axial deformation capacity of the SCED brace [4]. One $5350 \mathrm{~mm}$ long dual-core SCB was tested to evaluate its seismic performances; the results

Received May $1^{\text {st }}, 2014$, Accepted for publication September $16^{\text {th }}, 2014$.

Copyright (C) 2014 Published by ITB Journal Publisher, ISSN: 2337-5779, DOI: 10.5614/j.eng.technol.sci.2014.46.4.1 
are presented in this paper. The seismic demands on either the BRB or dual-core SCB's steel frames, under different ground motions, can be found elsewhere [5].

\section{Sandwiched Buckling-Restrained Brace}

A sandwiched BRB (Figure 1) is composed of a core plate and two identical restraining members, which are formed by welding a steel channel to a flat plate (face plate) and then filled with concrete or mortar. The benefit of using the proposed $\mathrm{BRB}$ is the ability to disassemble the brace, which not only means that the core plate can be replaced independently of the restraining members, but also provides an opportunity for inspection of the core.

The maximum compressive load is based on a limit state of BRB global stability and is estimated from equation [1]

$$
P_{\max , g}=\frac{M_{p}^{g}}{i+g+e+\frac{M_{p}^{g}}{P_{e}}}
$$

where $i$ is the initial imperfection at the center of the BRB, $g$ is the gap between the core plate and restraining member, $e$ is the eccentricity at the BRB end, $P_{e}$ is the Euler buckling load of the restraining member, and ${ }^{M_{p}^{g}}$ is the plastic moment capacity of two restraining members.

The test program consisted of cyclic tests of four BRBs. BRB 1 had the core length of $6550 \mathrm{~mm}$, and BRB 2-4 (Figure 1) had the core length of $1720 \mathrm{~mm}$, with a larger cross-sectional area than the BRB 1. BRBs 2 and 4 used a steel channel $180 \times 60 \times 4.5 \times 4.5$ and a flat plate to form a restraining member, which was filled with mortar. BRB 3 used a steel channel $180 \times 75 \times 4.5 \times 4.5$ and a flat plate to form a restraining member without using mortar inside the channel. After BRB 2 completed its tests, BRB 4 reused the restraining member of BRB 2 for subsequent tests. In this way, the performance of the existing-restraining member after replacing a new core can be examined. ASTM A572 GR.50 steel was specified for the core plate, side plate, and face plate. The specified 28-day mortar strength was $48 \mathrm{MPa}$. The ratio of $P_{\max , g} / P_{y}$ was 2.7-2.9 for BRBs 1-4, where $P_{y}$ is the core plate yield load. These values were larger than the suggested value of 2.5 [1], so no global buckling in BRBs 1-4 were expected before the core plate reached its ultimate compressive load, $P_{u}\left(=\beta F_{u} A_{c}\right)$ where $\beta$ is the compression strength adjustment factor and a value of 1.15 is used to estimate the maximum compressive force. Tensile strength $F_{u}$ was obtained from the material tensile coupon test. BRBs 1-3 were subjected to the prescribed loading protocol in Section T6 of AISC seismic provisions [6] until the specimens failed or a limit state of the test setup was reached. BRB 4 was 
tested using a near-field loading protocol and then an AISC loading protocol until failure.

\section{$2.1 \quad$ Test Results of BRBs}

Figure 2 shows the measured axial force versus axial displacement for only BRBs 3-4. BRB 3 was tested in the setup (Figure 1(a)) and exhibited stable hysteretic behaviors up to a maximum core strain of $3.8 \%$ (Figure 2). A fracture of the core plate for BRB 3 was observed during the third cycle, at a core strain of $3.8 \%$ (Figure 1(b)). The cumulative plastic ductility after the test was 856 for BRB 3, exceeding the value of 200 specified by AISC seismic provisions [6]. It indicates that the sandwiched BRB without concrete in the steel channel does not affect the performance of the BRB. BRB 4 reused the undamaged restraining member of BRB 2 and a new steel core. Figure 2 shows stable hysteretic behavior up to the maximum core strain of $2.5 \%$ in tension and $4.6 \%$ in compression, which was conducted based on a near-field loading protocol. No yielding or buckling of the restraining member was observed after the Phase I test.
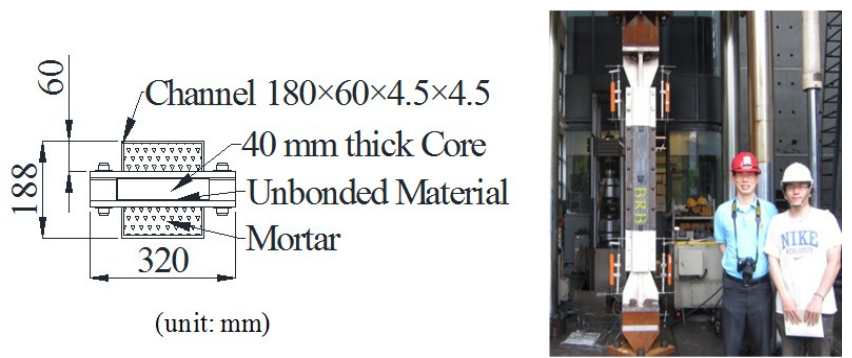

(a) BRBs 2-4

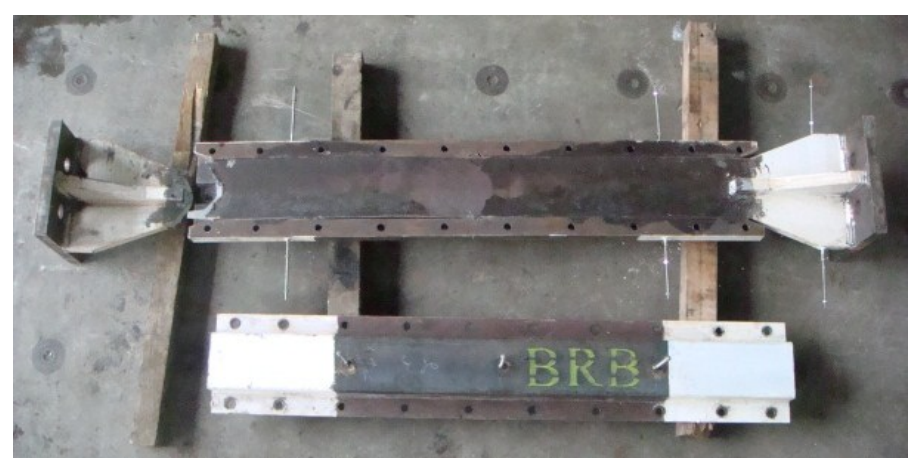

(b) Fracture (BRB 3, $\varepsilon_{\max }=3.8 \%$ )

Figure 1 BRB Size and failure mode. 
The sandwiched BRB has recently been used as earthquake-resisting members in Kaohsiung city library, Taiwan and Gansu science museum, China (Figure 3). These two new public buildings located in high-seismic areas of Taiwan and China, respectively, are under construction and will be completed in 2015. The axial capacity of the BRBs used in these two buildings ranges from $2000 \mathrm{kN}$ to $13000 \mathrm{kN}$.
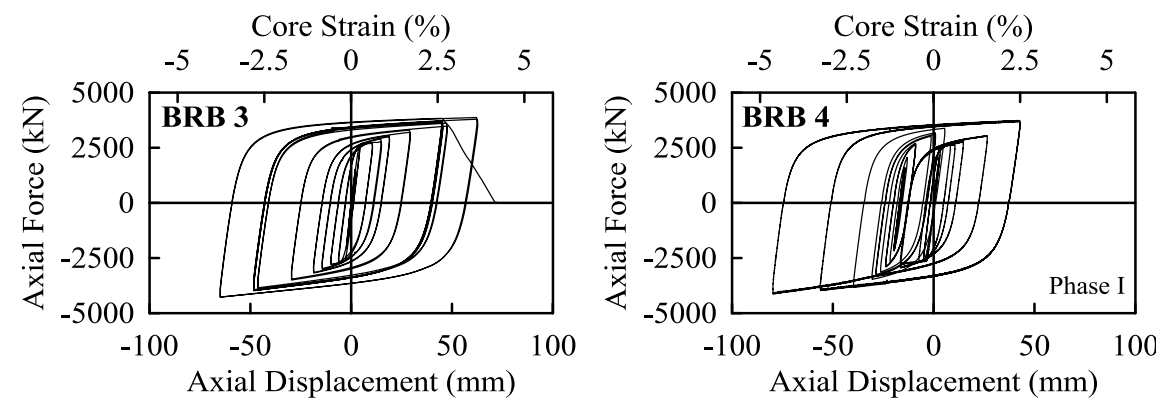

Figure 2 Hysteretic responses of BRBs.

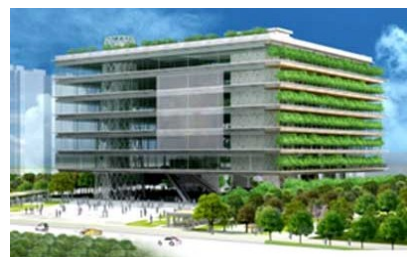

(a) Kaohsiung City Library (Taiwan)

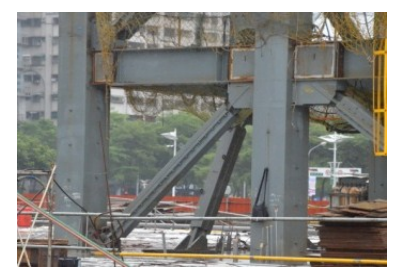

Figure 3 Application of sandwiched BRBs in Taiwan and China.

\section{Dual-Core Self-Centering Brace (SCB)}

Figure 4 presents the proposed dual-core SCB, which consists of three steel bracing members, two PT element sets, energy dissipation devices, and end plates. An energy dissipative device, which is located at one end of the brace, is activated by the relative motion is induced between the first core and outer box. All bracing members, end plates, and tendons in the dual-core SCB are arranged so that a relative motion is induced between these bracing members and causes serial elongation of the inner and outer tendons to achieve the desired brace elongation or shortening, which is always two times that of the tendon elongation.

\subsection{Kinematics and Mechanics}

Figure 5 presents the kinematics and hysteretic response of the dual-core SCB. Once the activation load, $F_{d t}$, of a dual-core SCB is exceeded, the inner end 
plate moves in the same direction with respect to the outer end plate, resulting in a brace deformation two times that of the tendon elongation $\delta$ (Figure 5(a)). The elongation in each tendon set $\delta$ causes the axial deformation of $2 \delta$ in the dual-core SCB. The brace returns to its original position when the load is removed (Figure 5(b)).

\section{Tests of the Dual-Core SCB}

The test program consisted of cyclic tests of one dual-core SCB specimen. The dual-core SCB had a first core of $\mathrm{H} 230 \times 210 \times 15 \times 15 \mathrm{~mm}$, two second cores of $\mathrm{T} 180 \times 180 \times 8 \mathrm{~mm}$, and an outer box tube of $\mathrm{T} 340 \times 440 \times 8 \mathrm{~mm}$. The specimen had eight $22 \mathrm{~mm}$-diameter E-glass fiber-reinforced-polymer (FRP) tendons as tensioning elements. The initial PT force in the brace was set to $260 \mathrm{kN}$. The friction devices placed on one end of the braces was set to produce friction forces of $250 \mathrm{kN}$. The specimen was fabricated by a local steel fabricator in Taiwan and post-tensioned and tested at the NCREE, Taiwan (Figure 6(a)).

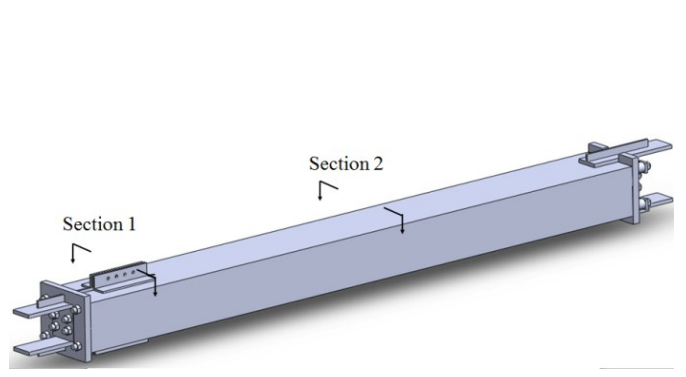

(a) Overall View

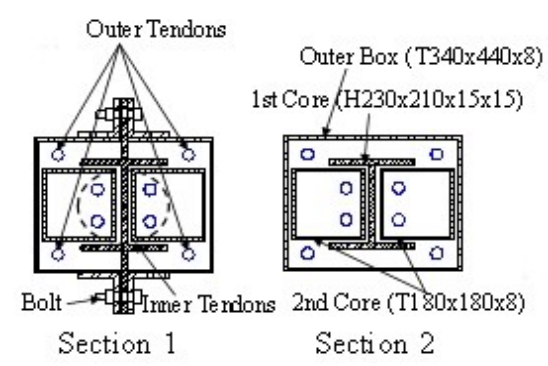

(b) Section View

Figure 4 A proposed dual-core SCB.

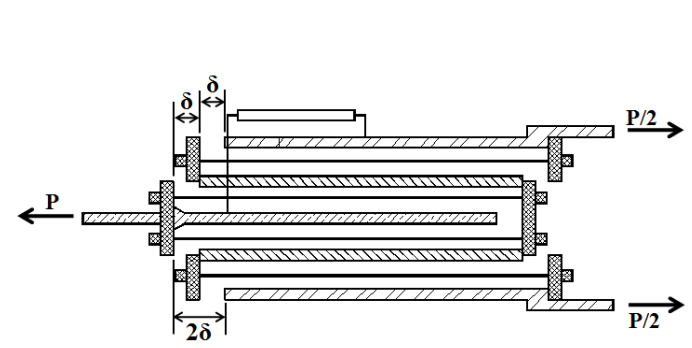

(a) Brace Kinematics

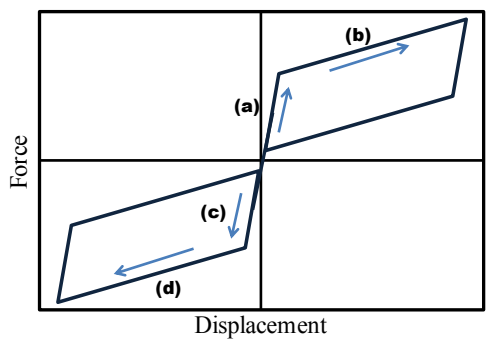

(b) Hysteretic Response

Figure 5 Kinematics and hysteretic response of the dual-core SCB. 


\subsection{Test Results}

The dual-core SCB specimen was subjected to four loading phases. The specimen was first tested to a drift of $0.5 \%$ in Phase 1 before stressing bolts in the friction device to evaluate the initial PT force. In the following loading phases, eight bolts were used to stress the friction device. The specimen was then subjected to the standard loading protocol (Phase 2) specified in Section T6 of the AISC seismic provisions [6] for evaluating the BRB performance. The specimen was subjected to an additional fifteen low-cycle fatigue loading at a column drift of $1.5 \%$ (Phase 3). Finally, the specimen was reloaded under the standard loading protocol beyond the target drift of $2 \%$ until failure (Phase 4 ).

The dual-core SCB in Phase 2 test developed a stable energy dissipation and self-centering property up to an interstory drift of $2 \%$. The specimen under fifteen low-cycle fatigue loading tests at a $1.5 \%$ drift also showed replicable responses with very minor differences in the hysteretic loops (Figure 6(b)). After a complete loading protocol in the Phase 3 test, the specimen was reloaded with AISC loading protocol to a drift of $2.5 \%$ (Phase 4). When the specimen was loaded in $2.5 \%$ drift cycles (Figure 6(a)), the tendon strain was $1.23 \%$, lower than its $1.47 \%$ capacity. Therefore, no tensioning elements were damaged during the test, and the maximum axial force in the brace was 1300 $\mathrm{kN}$ (Figure 6(c)).

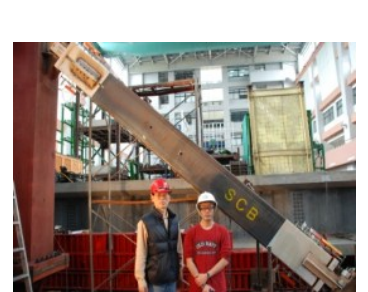

(a) $2.5 \% \mathrm{drift}$

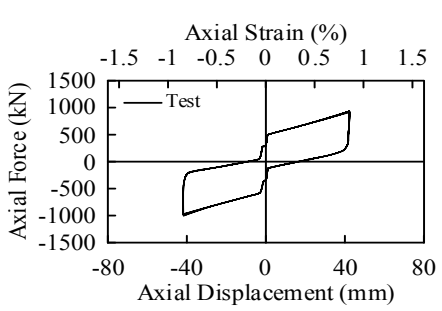

(b) Phase 3 (15 cycles)

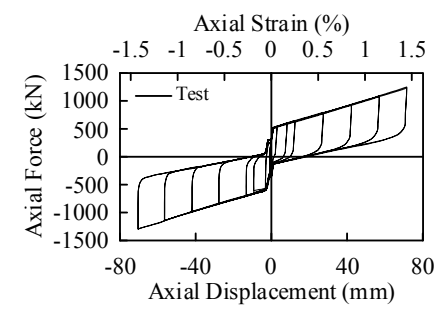

(c) Phase 4

Figure 6 Performance of the proposed dual-core SCB.

\section{Conclusions}

This work presents cyclic test results of the sandwiched buckling-restrained brace (BRB) and the dual-core self-centering brace (SCB). As long as the sandwiched BRB's design followed the design procedure [1], it maintained good seismic performance, up to a maximum compressive core strain of $4.6 \%$ in the cyclic loading. BRBs 2 and 3 had similar behaviors, indicating that concrete infill is not needed for the restraining member of the sandwiched BRB.

The dual-core SCB was developed to double the elongation capacity of the SCED brace and have the self-center response during the cyclic loading, 
eliminating the residual deformation as seen in the BRBs. The dual-core SCB specimen under cyclic loading showed a good self-centering hysteresis response, with a maximum interstory drift of $2.5 \%$.

\section{References}

[1] Chou, C.C. \& Chen, S.Y., Subassemblage Tests and Finite Element Analyses of Sandwiched Buckling-Restrained Braces, Engineering Structures, 32, pp. 2108-2121, 2010.

[2] Chou, C.C., Chen, Y.C. \& Chung, P.T., Dual-Core Self-Centering Energy Dissipation Brace Apparatus, No. 8316589 B2, US Patent and Trademark Office, USA, 2012.

[3] Chou, C.C. \& Chen, Y.C., Development of Steel Dual-Core SelfCentering Braces: Quasi-Static Cyclic Tests and Finite Element Analyses, Earthquake Spectra, http://earthquakespectra.org/toc/eqsa/0/0 (available online, September 2013).

[4] Christopoulos, C., Tremblay, R., Kim, H.J. \& Lacerte, M., Self-Centering Energy Dissipative Bracing System for the Seismic Resistance of Structures: Development and Validation, J. Structural Engineering, ASCE, 134(1), 96-107, 2008.

[5] Chou, C.C., Chen, Y.C., Pham, D.H. \& Truong, V.M., Steel Braced Frames with Dual-Core SCBS and Sandwiched BRBs: Mechanics, Modeling and Seismic Demands, Engineering Structures, 72, pp. 26-40, 2014.

[6] AISC, Seismic Provisions for Structural Steel Buildings, American Institute of Steel Construction, Chicago, IL. 2010. 